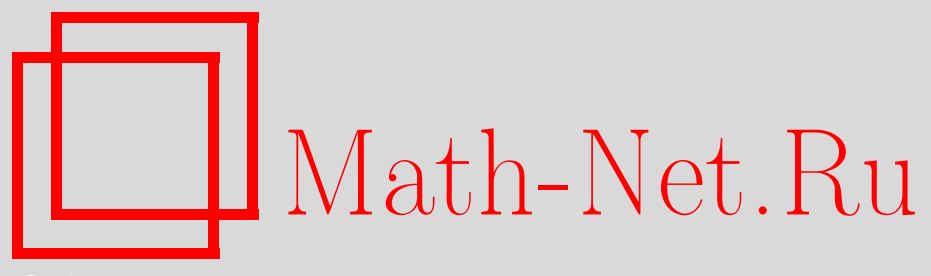

Б. В. Базалий, С. П. Дегтярев, Граничная задача для вырождающихся на границе области эллиптических уравнений в весовых пространствах Гёльдера, Матем. сб., 2013, том 204, номер 7, 25-46

DOI: https://doi.org/10.4213/sm8078

Использование Общероссийского математического портала Math-Net.Ru подразумевает, что вы прочитали и согласны с пользовательским соглашением http://www . mathnet.ru/rus/agreement

Параметры загрузки:

IP : 34.229 .108 .108

26 апреля 2023 г., 10:42:37

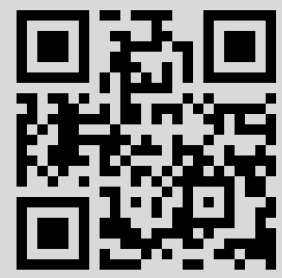




\author{
Б. В. Базалий, С. П. Дегтярев
}

\title{
Граничная задача для вырождающихся на границе области эллиптических уравнений в весовых пространствах Гёльдера
}

Изучается эллиптическая краевая задача для уравнений второго порядка с неотрицательной характеристической формой в условиях слабого вырождения на границе области. Получены априорные оценки решения и доказана разрешимость задачи в пространствах Гёльдера с весом.

Библиография: 18 названий.

Ключевые слова: вырождающееся эллиптическое уравнение, весовые пространства Гёльдера, априорная оценка.

DOI: $10.4213 / \operatorname{sm} 8078$

\section{§ 1. Введение}

Пусть $\alpha \in(0,1), \beta \in(0,1 / 2)$ таковы, что

$$
0<\beta<\min \{\alpha, 1-\alpha\} .
$$

Пусть $\Omega$ - ограниченная область в $\mathbb{R}^{N}$ с границей класса $C^{2+\beta}$; мы используем стандартные пространства Гёльдера $C^{2+\beta}$. Пусть $d(x)-$ функция класса $C^{1+\beta}(\bar{\Omega})$ такая, что

$$
\nu \operatorname{dist}(x, \partial \Omega) \leqslant d(x) \leqslant C \operatorname{dist}(x, \partial \Omega), \quad \nu>0
$$

здесь и всюду ниже через $\nu, C$ мы будем обозначать все абсолютные константы либо константы, зависящие только от раз и навсегда зафиксированных данных задачи.

Рассмотрим первую краевую задачу для искомой функции $u(x)$

$$
\begin{gathered}
L u \equiv d(x)^{\alpha} \sum_{i, j=1}^{N} a_{i j}(x) u_{x_{i} x_{j}}+\sum_{i=1}^{N} a_{i}(x) u_{x_{i}}+a(x) u=f(x), \quad x \in \Omega, \\
u(x)=\varphi(x), \quad x \in \partial \Omega .
\end{gathered}
$$

Здесь

$$
a_{i j}(x), a_{i}(x), a(x) \in C^{\beta}(\bar{\Omega})
$$

- заданные функции, причем для $\xi \in \mathbb{R}^{N}$

$$
\nu \xi^{2} \leqslant \sum_{i, j=1}^{N} a_{i j}(x) \xi_{i} \xi_{j} \leqslant C \xi^{2}, \quad x \in \bar{\Omega} .
$$


Мы предполагаем, что заданные функции $f(x)$ и $\varphi(x)$ обладают свойствами

$$
f(x) \in C^{\beta}(\bar{\Omega}), \quad \varphi(x) \in C^{2+\beta-\alpha}(\partial \Omega) .
$$

Краевая задача (1.3), (1.4) относится к числу задач для уравнения второго порядка с неотрицательной характеристической формой, имеющих к настоящему времени более чем полувековую историю интенсивных исследований. В книге О. А. Олейник и Е. В. Радкевича [1] приведен достаточно полный обзор работ в этой области вплоть до начала 70-х годов прошлого века. В частности, отмечается, что принципиальное значение в развитии теории для таких задач имела статья М. В. Келдыша [2]. Было обнаружено, что при достаточно сильном вырождении и определенных условиях на коэффициенты младших членов в уравнении, вырождающемся на границе области, граница может быть свободной от граничных условий. В работе [3] были сформулированы условия, при которых может быть поставлена первая краевая задача для уравнений вида (1.3); отметим, что эти условия выполнены при $\alpha \in(0,1)$.

Результаты о корректности краевых задач для вырождающихся эллиптических уравнений в пространствах Соболева можно найти в работах М. И. Вишика [4], М. И. Вишика и О. А. Ладыженской [5], О. А. Олейник [6], М. И. Вишика и В. В. Грушина [7].

Обзор литературы, посвященной более поздним исследованиям для граничных эллиптических задач с вырождением, можно найти в книге [8] (это второе, с изменениями, издание книги [1]), а также в книге [9].

Авторам известно небольшое число работ, в которых рассматриваемая задача изучается в пространствах Гёльдера. Эти работы относятся к случаю $\alpha=1$. Здесь мы укажем на работы [10]-[12], в которых при некоторых достаточных условиях на коэффициенты оператора $L$ показано, что $L$ осуществляет изоморфизм некоторых весовых пространств Гёльдера.

Целью настоящей работы является изучение задачи (1.3), (1.4) при $0<\alpha<1$ (слабое вырождение) в классах гладких функций, а именно в весовых пространствах Гёльдера.

Определим весовое банахово пространство функций $C_{\alpha}^{2+\beta}(\bar{\Omega})$ такое, что для $u(x) \in C_{\alpha}^{2+\beta}(\bar{\Omega})$ конечна норма

$$
|u|_{\alpha, \bar{\Omega}}^{(2+\beta)}=|u|_{\bar{\Omega}}^{(0)}+\sum_{i=1}^{N}\left|u_{x_{i}}\right|_{\bar{\Omega}}^{(\beta)}+\sum_{i, j=1}^{N}\left|d(x)^{\alpha} u_{x_{i} x_{j}}\right|_{\bar{\Omega}}^{(0)}+\sum_{i, j=1}^{N}\left\langle d(x)^{\alpha} u_{x_{i} x_{j}}\right\rangle_{x, \bar{\Omega}}^{(\beta)}
$$

где использованы обозначения

$$
|v|_{\bar{\Omega}}^{(0)}=\max _{\bar{\Omega}}|v(x)|, \quad|v|_{\bar{\Omega}}^{(\beta)}=|v|_{\bar{\Omega}}^{(0)}+\langle v\rangle_{x, \bar{\Omega}}^{(\beta)}
$$

$\langle v\rangle_{x, \bar{\Omega}}^{(\beta)}-$ константа Гёльдера функции $v$ с показателем $\beta$.

Отметим, что мы не указываем явно в определении пространства $C_{\alpha}^{2+\beta}(\bar{\Omega})$ и его нормы функцию $d(x)$, так как нормы, построенные по двум функциям $d(x)$ и $\widetilde{d}(x)$ из класса $C^{1+\beta}(\bar{\Omega})$, удовлетворяющим условию $(1.2)$, эквивалентны. 
Наряду с уравнением (1.3) рассмотрим уравнение

$$
L u-\lambda u=f,
$$

где $\lambda$ - комплексный параметр.

Сформулируем теперь основной результат, относящийся к эллиптической краевой задаче.

Теорема 1. Пусть выполнены условия (1.2), (1.1), (1.5)-(1.7), и пусть в (1.3) $a(x) \leqslant 0$. Тогда задача (1.3), (1.4) имеет единственное решение и $(x)$ из пространства $C_{\alpha}^{2+\beta}(\bar{\Omega})$, причем

$$
|u|_{\alpha, \bar{\Omega}}^{(2+\beta)} \leqslant C\left(|f|_{\bar{\Omega}}^{(\beta)}+|\varphi|_{\partial \Omega}^{(2+\beta-\alpha)}\right),
$$

a оператор задачи (1.3), (1.4) осуществляет изоморфизм пространства $C_{\alpha}^{2+\beta}(\bar{\Omega})$ на пространство $C^{\beta}(\bar{\Omega}) \times C^{2+\beta-\alpha}(\partial \Omega)$.

Если же в операторе (1.3) коэффичиент $а(x)$ произволен, то для задачи (1.9), (1.4) существует счетное число $\lambda_{k} \in \mathbb{C},\left|\lambda_{k}\right| \rightarrow \infty$ nри $k \rightarrow \infty$, таких, что при $\lambda=\lambda_{k}$ однородная задача (1.9), (1.4) имеет ненулевые решения, причем пространство таких решений конечномерно. В этом случае неоднородная задача (1.9), (1.4) разрешима при выполнении конечного числа условий на $f$ и $\varphi$, причем число этих условий совпадает с размерностъю ядра оператора задачи (1.9), (1.4). При $\lambda \neq \lambda_{k}$ оператор задачи (1.9), (1.4) осуществляет изоморфизм пространства $C_{\alpha}^{2+\beta}(\bar{\Omega})$ на пространство $C^{\beta}(\bar{\Omega}) \times C^{2+\beta-\alpha}(\partial \Omega)$, m.е., в частности, задача (1.9), (1.4) однозначно разрешима и справедлива оценка (1.10).

Кроме того, при любом $\lambda \in \mathbb{C}$ из единственности решения задачи (1.9), (1.4) следует ее разрешимость и оценка (1.10).

Статья состоит из шести параграфов. В 22 доказывается, что пространство следов на $\partial \Omega$ функций из класса $C_{\alpha}^{2+\beta}(\bar{\Omega})$ есть в точности пространство $C^{2+\beta-\alpha}(\partial \Omega)$. В $\S 3$ рассмотрена модельная задача в полупространстве для уравнения Лапласа с вырождением и получены точные коэрцитивные оценки решения в весовом пространстве Гёльдера. В $\S 4$ на основании результатов для модельной задачи приведена априорная оценка Шаудера решения задачи (1.3), (1.4). Для доказательства существования решения задачи (1.3), (1.4) в §5 рассмотрим разрешимость и оценки решений задач для уравнений с вырождением в областях специального вида. При этом сначала используется разрешимость в пространстве $W_{p}^{2}$, а затем повышается гладкость полученного решения. В $\S 6$ завершается доказательство теоремы 1.

\section{§ 2. Продолжение функций класса $C^{2+\beta-\alpha}(\partial \Omega)$ внутрь области и следы функций класса $C_{\alpha}^{2+\beta}(\bar{\Omega})$ на $\partial \Omega$}

Применяя технику локального распрямления границы (см., например, [13; гл. IV]), мы можем ограничиться рассмотрением случая, когда $\Omega$ представляет собой полупространство. 
Пусть $\mathbb{R}_{+}^{N}=\left\{x \in \mathbb{R}^{N}: x_{N}>0\right\}, \mathbb{R}^{N-1}=\left\{x \in \mathbb{R}^{N}: x_{N}=0\right\}$. Пусть на $\mathbb{R}^{N-1}$ задана финитная функция $\varphi(x) \in C^{2+\beta-\alpha}\left(\mathbb{R}^{N-1}\right)$. Покажем, что $\varphi(x)$ может быть продолжена в $\mathbb{R}_{+}^{N}$ до финитной функции класса $C_{\alpha}^{2+\beta}\left(\overline{\mathbb{R}_{+}^{N}}\right)$. При этом в определении нормы в пространстве $C_{\alpha}^{2+\beta}\left(\overline{\mathbb{R}_{+}^{N}}\right)$ роль весовой функции $d(x)$ в соотношении (1.8) играет функция $d(x)=x_{N}$.

Пусть $u(x)$ - решение задачи Дирихле

$$
\begin{gathered}
\Delta u(x)=0, \quad x \in \mathbb{R}_{+}^{N}, \\
u(x)=\varphi(x), \quad x_{N}=0 .
\end{gathered}
$$

В соответствии с $[14 ; \S 5.4]$, для мультииндексов $\bar{k}=\left(k_{1}, \ldots, k_{N}\right)$ таких, что $|\bar{k}|=k_{1}+\cdots+k_{N} \geqslant 2$ и $x_{N}<1$, выполнено

$$
\left|D^{\bar{k}} u\left(x^{\prime}, x_{N}\right)\right| \leqslant C_{k}|\varphi|_{\mathbb{R}^{N-1}}^{(2+\beta-\alpha)} x_{N}^{-|\bar{k}|+(2+\beta-\alpha)}, \quad x=\left(x^{\prime}, x_{N}\right) .
$$

Пусть $D^{2} u$ - некоторая вторая производная $u_{x_{i} x_{j}}$. Рассмотрим отдельно гёльдеровость $x_{N}^{\alpha} D^{2} u$ по переменным $x^{\prime}=\left(x_{1}, \ldots, x_{N-1}\right)$ и по $x_{N}$. Сначала рассмотрим гёльдеровость по $x^{\prime}$. Имеем

$$
\left\langle x_{N}^{\alpha} D^{2} u\right\rangle_{x^{\prime}, \overline{\mathbb{R}_{+}^{N}}}^{(\beta)}=x_{N}^{\alpha}\left\langle D^{2} u\right\rangle_{x^{\prime}, \overline{\mathbb{R}_{+}^{N}}}^{(\beta)} .
$$

В силу известного интерполяционного неравенства (см. [15; гл. 1])

$$
\left\langle D^{2} u\right\rangle_{x^{\prime}, \overline{\mathbb{R}_{+}^{N}}}^{(\beta)} \leqslant C\left(|u| \frac{(2)}{\mathbb{R}_{+}^{N}}\right)^{1-\beta}\left(|u| \frac{(3)}{\mathbb{R}_{+}^{N}}\right)^{\beta}
$$

и неравенства (2.3) из (2.4) получаем

$$
\begin{aligned}
\left\langle x_{N}^{\alpha} D^{2} u\right\rangle_{x^{\prime}, \overline{\mathbb{R}_{+}^{N}}}^{(\beta)} & \leqslant C x_{N}^{\alpha}|\varphi|_{\mathbb{R}^{N-1}}^{(2+\beta-\alpha)}\left(x_{N}^{-2+(2+\beta-\alpha)}\right)^{1-\beta}\left(x_{N}^{-3+(2+\beta-\alpha)}\right)^{\beta} \\
& =C x_{N}^{\alpha-2+(2+\beta-\alpha)-\beta}|\varphi|_{\mathbb{R}^{N-1}}^{(2+\beta-\alpha)}=C|\varphi|_{\mathbb{R}^{N-1}}^{(2+\beta-\alpha)} .
\end{aligned}
$$

Рассмотрим теперь гёльдеровость функции $x_{N}^{\alpha} D^{2} u$ по переменной $x_{N}$. Пусть $0<\bar{x}_{N}<x_{N}$. Рассмотрим два случая. Если $\left|x_{N}-\bar{x}_{N}\right| \geqslant x_{N} / 2$, то

$$
\begin{aligned}
& \frac{\left|x_{N}^{\alpha} D^{2} u(x)-\bar{x}_{N}^{\alpha} D^{2} u(\bar{x})\right|}{\left|x_{N}-\bar{x}_{N}\right|^{\beta}} \leqslant C\left\{\left|x_{N}^{\alpha-\beta} D^{2} u\left(x^{\prime}, x_{N}\right)\right|+\left|\bar{x}_{N}^{\alpha-\beta} D^{2} u\left(x^{\prime}, \bar{x}_{N}\right)\right|\right\} \\
& \quad \equiv C\left\{A_{1}+A_{2}\right\} .
\end{aligned}
$$

В силу (2.3)

$$
A_{1} \leqslant C|\varphi|_{\mathbb{R}^{N-1}}^{(2+\beta-\alpha)} x_{N}^{\alpha-\beta} x_{N}^{-2+(2+\beta-\alpha)}=C|\varphi|_{\mathbb{R}^{N-1}}^{(2+\beta-\alpha)}
$$

Оценка $A_{2}$ полностью аналогична.

Пусть теперь $\left|x_{N}-\bar{x}_{N}\right|<x_{N} / 2$. Обозначим $\Pi\left(x_{N}\right)=\left\{y \in \mathbb{R}_{+}^{N}:\left|y_{N}-x_{N}\right| \leqslant\right.$ $\left.x_{N} / 2\right\}$. Тогда в рассматриваемом случае

$$
\begin{aligned}
& \frac{\left|x_{N}^{\alpha} D^{2} u(x)-\bar{x}_{N}^{\alpha} D^{2} u(\bar{x})\right|}{\left|x_{N}-\bar{x}_{N}\right|^{\beta}} \leqslant C\left\langle y_{N}^{\alpha} D^{2} u\right\rangle_{y, \Pi\left(x_{N}\right)}^{(\beta)} \\
& \quad \leqslant C\left\{\left|y_{N}^{\alpha-\beta} D^{2} u\right|_{\Pi\left(x_{N}\right)}^{(0)}+x_{N}^{\alpha}\left\langle D^{2} u\right\rangle_{y, \Pi\left(x_{N}\right)}^{(\beta)}\right\} \equiv C\left\{A_{1}+A_{2}\right\} .
\end{aligned}
$$


При этом в силу $(2.3)$

$$
A_{1} \leqslant C x_{N}^{\alpha-\beta}\left|D^{2} u\right|_{\Pi\left(x_{N}\right)}^{(0)} \leqslant C|\varphi|_{\mathbb{R}^{N-1}}^{(2+\beta-\alpha)} x_{N}^{\alpha-\beta} x_{N}^{-2+(2+\beta-\alpha)}=C|\varphi|_{\mathbb{R}^{N-1}}^{(2+\beta-\alpha)} .
$$

Для оценки $A_{2}$ применим интерполяционное неравенство (2.5). Тогда в силу (2.3)

$$
\begin{aligned}
A_{2} & \leqslant C x_{N}^{\alpha}\left(|u|_{\Pi\left(x_{N}\right)}^{(2)}\right)^{1-\beta}\left(|u|_{\Pi\left(x_{N}\right)}^{(3)}\right)^{\beta} \\
& \leqslant C x_{N}^{\alpha}|\varphi|_{\mathbb{R}^{N-1}}^{(2+\beta-\alpha)}\left(x_{N}^{-2+(2+\beta-\alpha)}\right)^{1-\beta}\left(x_{N}^{-3+(2+\beta-\alpha)}\right)^{\beta}=C|\varphi|_{\mathbb{R}^{N-1}}^{(2+\beta-\alpha)} .
\end{aligned}
$$

Таким образом,

$$
\left\langle x_{N}^{\alpha} D^{2} u\right\rangle_{x, \mathbb{R}_{+}^{N}}^{(\beta)} \leqslant C|\varphi|_{\mathbb{R}^{N-1}}^{(2+\beta-\alpha)} .
$$

Совершенно аналогично доказывается, что

$$
\left|x_{N}^{\alpha} D^{2} u\right|_{\mathbb{R}_{+}^{N}}^{(0)} \leqslant C|\varphi|_{\mathbb{R}^{N-1}}^{(2-\alpha)} \leqslant C|\varphi|_{\mathbb{R}^{N-1}}^{(2+\beta-\alpha)} .
$$

Оценки же младших слагаемых в норме пространства $C_{\alpha}^{2+\beta}\left(\overline{\mathbb{R}_{+}^{N}}\right)$

$$
\left|u_{x_{i}}\right| \frac{(\beta)}{\mathbb{R}_{+}^{N}} \leqslant C|\varphi|_{\mathbb{R}^{N-1}}^{(2+\beta-\alpha)}
$$

следуют из хорошо известных оценок потенциала двойного слоя либо получаются так же, как и выше, в виде оценки

$$
|u|_{\mathbb{R}_{+}^{N}}^{(1+\beta)} \leqslant C|\varphi|_{\mathbb{R}^{N-1}}^{(1+\beta)} \leqslant C|\varphi|_{\mathbb{R}^{N-1}}^{(2+\beta-\alpha)}
$$

с использованием интерполяционного неравенства

$$
|u| \frac{(1+\beta)}{\mathbb{R}_{+}^{N}} \leqslant C\left(|u| \frac{(1)}{\mathbb{R}_{+}^{N}}\right)^{1-\beta}\left(|u| \frac{(2)}{\mathbb{R}_{+}^{N}}\right)^{\beta} .
$$

Следовательно,

$$
|u|_{\alpha, \mathbb{R}_{+}^{N}}^{(2+\beta)} \leqslant C|\varphi|_{\mathbb{R}^{N-1}}^{(2+\beta-\alpha)} .
$$

Умножая функцию $u(x)$ на гладкую финитную функцию $\eta(x)=\eta\left(x^{\prime}, x_{N}\right)$, равную единице в окрестности носителя функции $\varphi\left(x^{\prime}\right)$, получаем финитное продолжение в область $x_{N}>0$ функции $\varphi\left(x^{\prime}\right)$ до функции класса $C_{\alpha}^{2+\beta}\left(\overline{\mathbb{R}_{+}^{N}}\right)$, причем выполнено неравенство (2.11). Отсюда вытекает следующая теорема.

Теорема 2. Пусть $\varphi(x) \in C^{2+\beta-\alpha}(\partial \Omega)$. Тогда $\varphi$ может быть продолжена в область $\bar{\Omega}$ до функиии $\Phi(x) \in C_{\alpha}^{2+\beta}(\bar{\Omega})$, причем

$$
|\Phi|_{\alpha, \bar{\Omega}}^{(2+\beta)} \leqslant C|\varphi|_{\partial \Omega}^{(2+\beta-\alpha)} .
$$

Отметим, что в силу этой теоремы мы можем в задаче $(1.3),(1.4)$, не ограничивая общности, считать, что $\varphi(x) \equiv 0$.

Покажем теперь, что пространство следов на $\partial \Omega$ функций класса $C_{\alpha}^{2+\beta}(\bar{\Omega})$ есть в точности пространство $C^{2+\beta-\alpha}(\partial \Omega)$. Как и выше, мы можем использовать локальное распрямление границы области при рассмотрении локально определяемых классов $C_{\alpha}^{2+\beta}(\bar{\Omega})$. Поэтому достаточно ограничиться случаем полупространства, т.е. рассмотреть финитную функцию $u(x)$ в области $\mathbb{R}_{+}^{N}$ класса $C_{\alpha}^{2+\beta}\left(\overline{\mathbb{R}_{+}^{N}}\right)$ и ее след при $x_{N}=0$. 
Лемма 1. Пусть финитная функиия и $(x)$ принадлежит $C_{\alpha}^{2+\beta}\left(\overline{\mathbb{R}_{+}^{N}}\right)$. Тогда $v\left(x^{\prime}\right)=u\left(x^{\prime}, 0\right) \in C^{2+\beta-\alpha}\left(\mathbb{R}^{N-1}\right)$, причем

$$
\left|v\left(x^{\prime}\right)\right|_{C^{2+\beta-\alpha}\left(\mathbb{R}^{N-1}\right)}=\left|u\left(x^{\prime}, 0\right)\right|_{C^{2+\beta-\alpha}\left(\mathbb{R}^{N-1}\right)} \leqslant C|u|_{\alpha, \mathbb{R}_{+}^{N}}^{(2+\beta)} .
$$

ДокАзАтЕЛЬство. Очевидно, достаточно показать, что равномерно по $x_{N}$ для любой функции $w=u_{x_{i}}, i=\overline{1, N}$, выполнена оценка

$$
\langle w\rangle_{x^{\prime}, \mathbb{R}^{N-1}}^{(1+\beta-\alpha)} \leqslant C|u|_{\alpha, \mathbb{R}_{+}^{N}}^{(2+\beta)} .
$$

Итак, пусть $w=u_{x_{i}}, i=\overline{1, N}$. Чтобы доказать (2.14), достаточно (как следует из [16]) проверить, что для $h>0$

$$
\frac{\left|\Delta_{h, x^{\prime}}^{2} w\left(x_{N}\right)\right|}{h^{1+\beta-\alpha}} \leqslant C|u|_{\alpha, \frac{\mathbb{R}_{+}^{N}}{(2+\beta)}}^{2},
$$

где $\Delta_{h, x^{\prime}}^{2} w\left(x_{N}\right) \equiv \Delta_{h, x^{\prime}}^{2} w \equiv \Delta_{h, x^{\prime}}^{2} w\left(x^{\prime}, x_{N}\right)$ - вторая разность от функции $w$ по переменной $x_{j}, j=\overline{1, N-1}$, с шагом $h$, т.е.

$$
\Delta_{h, x^{\prime}}^{2} w\left(x_{N}\right)=w\left(x^{\prime}+\vec{j} h, x_{N}\right)-2 w\left(x^{\prime}, x_{N}\right)+w\left(x^{\prime}-\vec{j} h, x_{N}\right),
$$

где $\vec{j}$ - единичный вектор в плоскости $\mathbb{R}^{N-1}$.

Рассмотрим два случая. Пусть сначала

$$
h \leqslant x_{N}
$$

Тогда для некоторых $\theta_{1}, \theta_{2} \in(0,1)$ по теореме о среднем получаем

$$
\begin{aligned}
\frac{\left|\Delta_{h, x^{\prime}}^{2} w\left(x_{N}\right)\right|}{h^{1+\beta-\alpha}} & \leqslant \frac{\left|x_{N}^{\alpha} \Delta_{h, x^{\prime}}^{2} w\left(x_{N}\right)\right|}{h^{1+\beta}} \\
& =\left|x_{N}^{\alpha} \frac{w_{x_{j}}\left(x^{\prime}+\vec{j} \theta_{1} h, x_{N}\right)-w_{x_{j}}\left(x^{\prime}-\vec{j} \theta_{2} h, x_{N}\right) \mid}{h^{\beta}}\right| \\
& =\frac{\left|x_{N}^{\alpha} u_{x_{i} x_{j}}\left(x^{\prime}+\vec{j} \theta_{1} h, x_{N}\right)-x_{N}^{\alpha} u_{x_{i} x_{j}}\left(x^{\prime}+\vec{j} \theta_{2} h, x_{N}\right)\right|}{h^{\beta}} \\
& \leqslant C\left\langle x_{N}^{\alpha} u_{x_{i} x_{j}}\right\rangle_{x^{\prime}, \overline{\mathbb{R}_{+}^{N}}}^{(\beta)} \leqslant C|u|_{\alpha, \overline{\mathbb{R}_{+}^{N}}}^{(2+\beta)} .
\end{aligned}
$$

Пусть теперь $h \geqslant x_{N}$. Представим разность $\Delta_{h, x^{\prime}}^{2} w\left(x_{N}\right)$ в виде

$$
\begin{aligned}
\Delta_{h, x^{\prime}}^{2} w\left(x_{N}\right) & =-\Delta_{h, x^{\prime}}^{2}\left(w\left(x^{\prime}, x_{N}+h\right)-w\left(x^{\prime}, x_{N}\right)\right)+\Delta_{h, x^{\prime}}^{2} w\left(x^{\prime}, x_{N}+h\right) \\
& \equiv A_{1}+A_{2} .
\end{aligned}
$$

В силу того, что для $A_{2}$ выполнено условие $(2.17)$, т.е. $h \leqslant x_{N}+h$, аналогично (2.18) получаем

$$
\frac{\left|A_{2}\right|}{h^{1+\beta-\alpha}} \leqslant C|u|_{\alpha, \overline{\mathbb{R}_{+}^{N}}}^{(2+\beta)} .
$$


Для оценки величины $\left|A_{1}\right| / h^{1+\beta-\alpha}$ воспользуемся соотношением

$$
w\left(x^{\prime}, x_{N}+h\right)-w\left(x^{\prime}, x_{N}\right)=h \int_{0}^{1} w_{x_{N}}\left(x^{\prime}, x_{N}+\theta h\right) d \theta,
$$

и поскольку $w_{x_{N}}=u_{x_{i} x_{N}}$, то

$$
\begin{aligned}
\frac{\left|A_{1}\right|}{h^{1+\beta-\alpha}} & \leqslant \int_{0}^{1} \frac{h^{\alpha}}{\left(x_{N}+\theta h\right)^{\alpha}}\left|\frac{\Delta_{h, x^{\prime}}^{2}\left(x_{N}+\theta h\right)^{\alpha} u_{x_{i} x_{N}}\left(x^{\prime}, x_{N}+\theta h\right)}{h^{\beta}}\right| d \theta \\
& \leqslant C\left\langle x_{N}^{\alpha} u_{x_{i} x_{N}}\right\rangle_{x^{\prime}, \mathbb{R}_{+}^{N}}^{(\beta)} \int_{0}^{1}\left(\frac{x_{N}}{h}+\theta\right)^{-\alpha} d \theta \leqslant C|u|_{\alpha, \mathbb{R}_{+}^{N}}^{(2+\beta)}
\end{aligned}
$$

Из (2.18), (2.20) и (2.21) следует (2.15), а следовательно, и (2.13).

Следствием этой леммы является следующая теорема.

Теорема 3. Пусть $u(x) \in C_{\alpha}^{2+\beta}(\bar{\Omega})$. Тогда $\left.\varphi \equiv u\right|_{\partial \Omega} \in C^{2+\beta-\alpha}(\partial \Omega)$, причем

$$
|\varphi|_{\partial \Omega}^{(2+\beta-\alpha)} \leqslant|u|_{\alpha, \bar{\Omega}}^{(2+\beta)}
$$

\section{§ 3. Модельная задача в полупространстве: оценки объемного потенциала}

Пусть функция $u(x)$ в области $\mathbb{R}_{+}^{N}=\left\{\left(x^{\prime}, x_{N}\right): x_{N}>0\right\}$ есть решение следующей модельной задачи:

$$
\begin{gathered}
x_{N}^{\alpha} \Delta u(x)=f(x), \quad x \in \mathbb{R}_{+}^{N}, \\
u\left(x^{\prime}, 0\right)=0,
\end{gathered}
$$

где заданная функция $f(x)$ принадлежит пространству $C^{\beta}\left(\overline{\mathbb{R}_{+}^{N}}\right), \operatorname{supp} f \subset B_{R}^{+}=$ $\overline{\mathbb{R}_{+}^{N}} \cap\{|x| \leqslant R\}$. Как хорошо известно, функция $u(x)$ может быть представлена в виде

$$
u(x)=\int_{\mathbb{R}_{+}^{N}} G(x, \xi) \xi_{N}^{-\alpha} f(\xi) d \xi
$$

где

$$
\begin{aligned}
G(x, \xi)=C & \left\{\left[\left(x^{\prime}-\xi^{\prime}\right)^{2}+\left(x_{N}-\xi_{N}\right)^{2}\right]^{-(N-2) / 2}\right. \\
& \left.-\left[\left(x^{\prime}-\xi^{\prime}\right)^{2}+\left(x_{N}+\xi_{N}\right)^{2}\right]^{-(N-2) / 2}\right\},
\end{aligned}
$$

причем для $G(x, \xi)$ справедлива оценка

$$
\left|D_{x}^{\overline{k_{1}}} D_{\xi}^{\overline{k_{2}}} G(x, \xi)\right| \leqslant C_{\bar{k}}|x-\xi|^{-N+2-\left|\overline{k_{1}}\right|-\left|\overline{k_{2}}\right|} .
$$

Цель этого параграфа - получить оценку нормы функции $u(x)$ в пространстве $C_{\alpha}^{2+\beta}\left(\overline{\mathbb{R}_{+}^{N}}\right)$ в терминах нормы правой части $|f|_{\mathbb{R}_{+}^{N}}^{(\beta)}$. 
Пусть $i, j=\overline{1, N}$ и $i, j$ не равны $N$ одновременно. Из оценки (3.5) и того, что $\alpha \in(0,1)$, следует, что потенциал (3.3) можно один раз дифференцировать по $x_{i}$, т.е.

$$
u_{x_{i}}=\int_{\mathbb{R}_{+}^{N}} G_{x_{i}}(x, \xi) \xi_{N}^{-\alpha} f(\xi) d \xi=\int_{\mathbb{R}_{+}^{N} \cap\{|\xi|<2 R\}} G_{x_{i}}(x, \xi) \xi_{N}^{-\alpha} f(\xi) d \xi,
$$

так как $f$ финитна. Получим представление для второй производной $u_{x_{i} x_{j}}$, считая, что $x_{N}>0, j \neq N$. Обозначим для $0<\varepsilon<x_{N}$

$$
u_{x_{i} \varepsilon}=\int_{\mathbb{R}_{+}^{N} \cap\{|\xi|<2 R\} \cap\{|x-\xi|>\varepsilon\}} G_{x_{i}}(x, \xi) \xi_{N}^{-\alpha} f(\xi) d \xi .
$$

Отметим, что $u_{x_{i} \varepsilon}$ равномерно на компактах сходится к $u_{x_{i}}$ при $\varepsilon \rightarrow 0$. Рассмотрим

$$
\begin{aligned}
\left(u_{x_{i} \varepsilon}\right)_{x_{j}}= & \int_{\mathbb{R}_{+}^{N} \cap\{|\xi|<2 R\} \cap\{|x-\xi|>\varepsilon\}} G_{x_{i} x_{j}}(x, \xi) \xi_{N}^{-\alpha} f(\xi) d \xi \\
& +\int_{|x-\xi|=\varepsilon} G_{x_{i}}(x, \xi) n_{j} \xi_{N}^{-\alpha} f(\xi) d S_{\xi} \equiv A_{1}+A_{2},
\end{aligned}
$$

где $n_{j}-j$-я компонента внешней нормали к сфере $\{|x-\xi|=\varepsilon\}$. Найдем предел при $\varepsilon \rightarrow 0$ величины $A_{2}$. Имеем

$$
\begin{aligned}
A_{2}=x_{N}^{-\alpha} f(x) \int_{|x-\xi|=\varepsilon} G_{x_{i}}(x, \xi) n_{j} d S_{\xi} \\
\quad+\int_{|x-\xi|=\varepsilon} G_{x_{i}}(x, \xi) n_{j}\left[\xi_{N}^{-\alpha} f(\xi)-x_{N}^{-\alpha} f(x)\right] d S_{\xi} \equiv A_{21}+A_{22},
\end{aligned}
$$

причем в силу (3.5) $A_{22} \rightarrow 0$ при $\varepsilon \rightarrow 0$.

Для вычисления предела $A_{21}$ при $\varepsilon \rightarrow 0$ отметим, что

$$
G_{x_{i}}(x, \xi)=C \frac{x_{i}-\xi_{i}}{|x-\xi|^{N}}+F(x, \xi),
$$

где $F(x, \xi)$ ограничена при фиксированном $x_{N}>0$ и в то же время $n_{j}=$ $\left(x_{j}-\xi_{j}\right) /|x-\xi|$. Поэтому

$$
\lim _{\varepsilon \rightarrow 0} A_{21}=C \lim _{\varepsilon \rightarrow 0} x_{N}^{-\alpha} f(x) \int_{|x-\xi|=\varepsilon} \frac{\left(x_{i}-\xi_{i}\right)\left(x_{j}-\xi_{j}\right)}{|x-\xi|^{N+1}} d S_{\xi}=C \delta_{i j} x_{N}^{-\alpha} f(x),
$$

где $\delta_{i j}-$ символ Кронекера.

Обратимся теперь к слагаемому $A_{1}$. Имеем

$$
\begin{aligned}
A_{1}= & \int_{\mathbb{R}_{+}^{N} \cap\{|\xi|<2 R\} \cap\{|x-\xi|>\varepsilon\}} G_{x_{i} x_{j}}(x, \xi) \xi_{N}^{-\alpha}[f(\xi)-f(x)] d \xi \\
& +f(x) \int_{\mathbb{R}_{+}^{N} \cap\{|\xi|<2 R\} \cap\{|x-\xi|>\varepsilon\}} G_{x_{i} x_{j}}(x, \xi) \xi_{N}^{-\alpha} d \xi \equiv A_{11}+A_{12} .
\end{aligned}
$$

Рассмотрим величину $A_{12}$. Так как $j \neq N$, то

$$
G_{x_{i} x_{j}}(x, \xi)=-G_{x_{i} \xi_{j}}(x, \xi) .
$$


Поэтому интегрирование по $\xi_{j}$ дает

$$
A_{12}=f(x)\left[\int_{\mathbb{R}_{+}^{N} \cap\{|\xi|=2 R\}} G_{x_{i}}(x, \xi) n_{j} \xi_{N}^{-\alpha} d S_{\xi}+\int_{|x-\xi|=\varepsilon} G_{x_{i}}(x, \xi) n_{j} \xi_{N}^{-\alpha} d S_{\xi}\right],
$$

где мы учли, что на поверхности $\left\{\xi_{N}=0\right\}$ выполнено $n_{j}=0$, так как $j \neq N$.

Переходя в $(3.9)$ к пределу при $\varepsilon \rightarrow 0$, как и в случае выражения $A_{21}$, получаем

$$
\lim _{\varepsilon \rightarrow 0} A_{12}=f(x) \int_{\mathbb{R}_{+}^{N} \cap\{|\xi|=2 R\}} G_{x_{i}}(x, \xi) n_{j} \xi_{N}^{-\alpha} d S_{\xi}+C \delta_{i j} x_{N}^{-\alpha} f(x) .
$$

Так как подынтегральное выражение в $A_{11}$ интегрируемо при $\varepsilon=0$ в силу гёльдеровости функции $f(\xi)$, то, переходя к пределу при $\varepsilon \rightarrow 0$, получим

$$
\lim _{\varepsilon \rightarrow 0} A_{11}=\int_{\mathbb{R}_{+}^{N} \cap\{|\xi|<2 R\}} G_{x_{i} x_{j}}(x, \xi) \xi_{N}^{-\alpha}[f(\xi)-f(x)] d \xi .
$$

Объединяя (3.8)-(3.11), окончательно получаем

$$
\begin{aligned}
u_{x_{i} x_{j}}= & \int_{\mathbb{R}_{+}^{N} \cap\{|\xi|<2 R\}} G_{x_{i} x_{j}}(x, \xi) \xi_{N}^{-\alpha}[f(\xi)-f(x)] d \xi \\
& +C \delta_{i j} x_{N}^{-\alpha} f(x)+f(x) \int_{\mathbb{R}_{+}^{N} \cap\{|\xi|=2 R\}} G_{x_{i}}(x, \xi) n_{j} \xi_{N}^{-\alpha} d S_{\xi} \\
\equiv & B_{1}(x)+B_{2}(x)+B_{3}(x) .
\end{aligned}
$$

Рассмотрим для переменных $x$ только область $B_{R}^{+}=\overline{\mathbb{R}_{+}^{N}} \cap\{|x| \leqslant R\}$. На этом множестве при $|\xi|=2 R$ функция $G_{x_{i}}(x, \xi)$ принадлежит $C_{x, \xi}^{\infty}$, и поскольку $\beta<\alpha$, то

$$
\left|x_{N}^{\alpha} B_{3}(x)\right| \frac{(\beta)}{B_{R}^{+}} \leqslant C_{R}|f| \frac{(\beta)}{\mathbb{R}_{+}^{N}} .
$$

Очевидно также, что

$$
\left|x_{N}^{\alpha} B_{2}(x)\right| \frac{(\beta)}{B_{R}^{+}} \leqslant C_{R}|f|_{\mathbb{R}_{+}^{N}}^{(\beta)} .
$$

Поэтому достаточно рассмотреть выражение

$$
v(x)=x_{N}^{\alpha} B_{1}(x)=x_{N}^{\alpha} \int_{\mathbb{R}_{+}^{N} \cap\{|\xi|<2 R\}} G_{x_{i} x_{j}}(x, \xi) \xi_{N}^{-\alpha}[f(\xi)-f(x)] d \xi .
$$

Оценим сначала $|v(x)| \frac{(0)}{B_{R}^{+}}$. Так как $|f(\xi)-f(x)| \leqslant|f| \frac{(\beta)}{\mathbb{R}_{+}^{N}}|x-\xi|^{\beta}$, то

$$
|v(x)| \leqslant C|f| \frac{(\beta)}{\mathbb{R}_{+}^{N}} x_{N}^{\alpha} \int_{B_{2 R}^{+}}\left[\left(x^{\prime}-\xi^{\prime}\right)^{2}+\left(x_{N}-\xi_{N}\right)^{2}\right]^{-(N-\beta) 2} \xi_{N}^{-\alpha} d \xi .
$$

Совершая в интеграле замену $x^{\prime}-\xi^{\prime}=\eta\left|x_{N}-\xi_{N}\right|$, получаем

$$
\begin{aligned}
|v(x)| & \leqslant C|f|_{\frac{\mathbb{R}_{+}^{N}}{(\beta)}} x_{N}^{\alpha} \int_{0}^{2 R} \xi_{N}^{-\alpha}\left|x_{N}-\xi_{N}\right|^{-1+\beta} d \xi_{N} \int_{|\eta|<2 R /\left|x_{N}-\xi_{N}\right|}\left(\eta^{2}+1\right)^{-(N+\beta) / 2} d \eta \\
& \leqslant C|f|_{\frac{\mathbb{R}_{+}^{N}}{(\beta)}} x_{N}^{\alpha} \int_{0}^{2 R} \xi_{N}^{-\alpha}\left|x_{N}-\xi_{N}\right|^{-1+\beta} d \xi_{N} .
\end{aligned}
$$


Оценка последнего интеграла с помощью замены $\xi_{N}=z x_{N}$ с учетом $0<\beta<$ $\alpha<1$ дает

$$
|v(x)| \leqslant C|f| \frac{(\beta)}{\mathbb{R}_{+}^{N}} x_{N}^{\alpha} x_{N}^{-\alpha-1+\beta+1} \int_{0}^{2 R / x_{N}} z^{-\alpha}|1-z|^{-1+\beta} d z \leqslant C|f| \frac{(\beta)}{\mathbb{R}_{+}^{N}} x_{N}^{\beta} .
$$

Перейдем теперь к оценке константы Гёльдера функции $v(x)$. При этом рассмотрим отдельно гёльдеровость $v(x)$ по переменной $x_{N}$ и по переменным $x^{\prime}$. Начнем с переменной $x_{N}$. Пусть $\varepsilon_{0} \in(0,1 / 4)$ фиксировано, и пусть $0<$ $\bar{x}_{N}<x_{N}$. Мы можем считать, не ограничивая общности, что

$$
\Delta x \equiv\left(x_{N}-\bar{x}_{N}\right) \leqslant \varepsilon_{0} x_{N} .
$$

Действительно, в противном случае

$$
\frac{\left|v\left(x^{\prime}, x_{N}\right)-v\left(x^{\prime}, \bar{x}_{N}\right)\right|}{\left|x_{N}-\bar{x}_{N}\right|^{\beta}} \leqslant \varepsilon_{0}^{-\beta}\left(\frac{\left|v\left(x^{\prime}, x_{N}\right)\right|}{\left|x_{N}\right|^{\beta}}+\frac{\left|v\left(x^{\prime}, \bar{x}_{N}\right)\right|}{\left|\bar{x}_{N}\right|^{\beta}}\right) \leqslant 2 \varepsilon_{0}^{-\beta} C|f| \frac{(\beta)}{\mathbb{R}_{+}^{N}},
$$

как это следует из оценки (3.16). Отметим также, что при условии $\left(x_{N}-\bar{x}_{N}\right) \leqslant$ $\varepsilon_{0} x_{N}$ величины $x_{N}$ и $\bar{x}_{N}$ эквивалентны, так как $1-\varepsilon_{0}<\bar{x}_{N} / x_{N}<1$.

Пусть $x=\left(x^{\prime}, x_{N}\right), \bar{x}=\left(x^{\prime}, \bar{x}_{N}\right)$. Рассмотрим разность

$$
\begin{aligned}
v(\bar{x}) & -v(x)=\left(\bar{x}_{N}^{\alpha}-x_{N}^{\alpha}\right) \int_{\mathbb{R}_{+}^{N} \cap\{|\xi|<2 R\}} G_{x_{i} x_{j}}(\bar{x}, \xi) \xi_{N}^{-\alpha}[f(\xi)-f(\bar{x})] d \xi \\
& +x_{N}^{\alpha}\left\{\int_{\mathbb{R}_{+}^{N} \cap\{|\xi|<2 R\}} G_{x_{i} x_{j}}(\bar{x}, \xi) \xi_{N}^{-\alpha}[f(\xi)-f(\bar{x})] d \xi\right. \\
& \left.-\int_{\mathbb{R}_{+}^{N} \cap\{|\xi|<2 R\}} G_{x_{i} x_{j}}(x, \xi) \xi_{N}^{-\alpha}[f(\xi)-f(x)] d \xi\right\} \equiv \mathcal{A}_{1}+\mathcal{A}_{2} .
\end{aligned}
$$

Поскольку

$$
\left|\bar{x}_{N}^{\alpha}-x_{N}^{\alpha}\right| \leqslant C \bar{x}_{N}^{\alpha-\beta}\left|\bar{x}_{N}-x_{N}\right|^{\beta},
$$

то из ранее полученной оценки (3.16) следует

$$
\begin{aligned}
\left|\mathcal{A}_{1}\right| & \leqslant C(\Delta x)^{\beta} \bar{x}_{N}^{-\beta}\left|\bar{x}_{N}^{\alpha} \int_{\mathbb{R}_{+}^{N} \cap\{|\xi|<2 R\}} G_{x_{i} x_{j}}(\bar{x}, \xi) \xi_{N}^{-\alpha}[f(\xi)-f(\bar{x})] d \xi\right| \\
& \leqslant C|f| \frac{(\beta)}{\mathbb{R}_{+}^{N}}(\Delta x)^{\beta} .
\end{aligned}
$$

Величину $\mathcal{A}_{2}$ представим в виде

$$
\begin{aligned}
\mathcal{A}_{2}=x_{N}^{\alpha} & \int_{B_{2 R}^{+} \cap\{|x-\xi|<2 \Delta x\}} G_{x_{i} x_{j}}(\bar{x}, \xi) \xi_{N}^{-\alpha}[f(\xi)-f(\bar{x})] d \xi \\
& -x_{N}^{\alpha} \int_{B_{2 R}^{+} \cap\{|x-\xi|<2 \Delta x\}} G_{x_{i} x_{j}}(x, \xi) \xi_{N}^{-\alpha}[f(\xi)-f(x)] d \xi \\
& +x_{N}^{\alpha} \int_{B_{2 R}^{+} \cap\{|x-\xi|>2 \Delta x\}} G_{x_{i} x_{j}}(\bar{x}, \xi) \xi_{N}^{-\alpha}\{[f(\xi)-f(\bar{x})]-[f(\xi)-f(x)]\} d \xi \\
& +x_{N}^{\alpha} \int_{B_{2 R}^{+} \cap\{|x-\xi|>2 \Delta x\}}\left[G_{x_{i} x_{j}}(\bar{x}, \xi)-G_{x_{i} x_{j}}(x, \xi)\right] \xi_{N}^{-\alpha}[f(\xi)-f(x)] d \xi \\
\equiv I_{1} & +I_{2}+I_{3}+I_{4} .
\end{aligned}
$$


При оценке $I_{1}$ заметим, что на множестве интегрирования величины $x_{N}$ и $\xi_{N}$ эквивалентны. Действительно,

$$
\left(1-2 \varepsilon_{0}\right) x_{N} \leqslant x_{N}-2 \Delta x \leqslant \xi_{N} \leqslant x_{N}+2 \Delta x \leqslant\left(1+2 \varepsilon_{0}\right) x_{N} .
$$

Кроме того, $\left(B_{2 R}^{+} \cap\{|x-\xi|<2 \Delta x\}\right) \subset\left(B_{2 R}^{+} \cap\{|\bar{x}-\xi|<3 \Delta x\}\right)$, так что для $I_{1}$ в силу (3.5) имеем

$$
\begin{aligned}
\left|I_{1}\right| & \leqslant C|f| \frac{(\beta)}{\mathbb{R}_{+}^{N}} x_{N}^{\alpha} \int_{|\bar{x}-\xi|<3 \Delta x}|\bar{x}-\xi|^{-N} x_{N}^{-\alpha}|\bar{x}-\xi|^{\beta} d \xi \\
& \leqslant C|f| \frac{(\beta)}{\mathbb{R}_{+}^{N}} \int_{|\bar{x}-\xi|<3 \Delta x}|\bar{x}-\xi|^{-N+\beta} d \xi=C|f|_{\frac{\mathbb{R}_{+}^{N}}{(\beta)}}(\Delta x)^{\beta} .
\end{aligned}
$$

Интеграл $I_{2}$ оценивается аналогично:

$$
\left|I_{2}\right| \leqslant C|f|_{\frac{\mathbb{R}_{+}^{N}}{(\beta)}}(\Delta x)^{\beta} .
$$

Интеграл $I_{3}$ перепишем в виде

$$
I_{3}=[f(x)-f(\bar{x})] x_{N}^{\alpha} \int_{B_{2 R}^{+} \cap\{|x-\xi|>2 \Delta x\}} G_{x_{i} x_{j}}(\bar{x}, \xi) \xi_{N}^{-\alpha} d \xi \equiv[f(x)-f(\bar{x})] A_{3} .
$$

Так как $j \neq N$, то $G_{x_{i} x_{j}}(\bar{x}, \xi)=-G_{x_{i} \xi_{j}}(\bar{x}, \xi)$, и, следовательно, $A_{3}$ можно представить в форме

$$
\begin{aligned}
A_{3}=x_{N}^{\alpha} & \int_{B_{2 R}^{+} \cap\{|\xi|=2 R\}} G_{x_{i}}(\bar{x}, \xi) n_{j} \xi_{N}^{-\alpha} d S_{\xi} \\
& +x_{N}^{\alpha} \int_{B_{2 R}^{+} \cap\{|x-\xi|=2 \Delta x\}} G_{x_{i}}(\bar{x}, \xi) n_{j} \xi_{N}^{-\alpha} d S_{\xi} \equiv A_{31}+A_{32},
\end{aligned}
$$

где мы учли, что компонента нормали $n_{j}$ тождественно равна нулю при $\xi_{N}=0$, так как $j \neq N$. Для оценки $A_{31}$ заметим, что так как мы рассматриваем случай $|x| \leqslant R$, то $\left|G_{x_{i}}(\bar{x}, \xi)\right| \leqslant C_{R}$ на множестве интегрирования в $A_{31}$. Поэтому

$$
\left|A_{31}\right| \leqslant C_{R} \int_{B_{2 R}^{+} \cap\{|\xi|=2 R\}} \xi_{N}^{-\alpha} d S_{\xi} \leqslant C_{R}
$$

Что же касается $A_{32}$, то в силу эквивалентности $x_{N}$ и $\xi_{N}$ на множестве интегрирования имеем

$$
\left|A_{32}\right| \leqslant C \int_{|x-\xi|=2 \Delta x}|\bar{x}-\xi|^{-(N-1)} d S_{\xi}
$$

Заметим, что на множестве $|x-\xi| \geqslant 2 \Delta x$ величины $|\bar{x}-\xi|$ и $|x-\xi|$ эквивалентны вследствие неравенств

$$
\begin{gathered}
|\bar{x}-\xi| \leqslant|x-\xi|+\Delta x \leqslant|x-\xi|+\frac{1}{2}|x-\xi|=\frac{3}{2}|x-\xi|, \\
|x-\xi| \leqslant|\bar{x}-\xi|+\Delta x \leqslant|\bar{x}-\xi|+\frac{1}{2}|x-\xi|
\end{gathered}
$$

так что

$$
|x-\xi| \leqslant 2|\bar{x}-\xi|
$$


Поэтому

$$
\left|A_{32}\right| \leqslant C \int_{|x-\xi|=2 \Delta x}|x-\xi|^{-(N-1)} d S_{\xi}=C .
$$

Из (3.23) и (3.24) следует оценка

$$
\left|I_{3}\right| \leqslant C_{R}|f(x)-f(\bar{x})| \leqslant C_{R}|f| \frac{(\beta)}{\mathbb{R}_{+}^{N}}(\Delta x)^{\beta} .
$$

Для оценки $I_{4}$ воспользуемся теоремой о среднем, что дает

$$
\left|I_{4}\right| \leqslant C|f|_{\frac{\mathbb{R}_{+}^{N}}{(\beta)}}^{\alpha} x_{N}^{\alpha}|\Delta x| \int_{B_{2 R}^{+} \cap\{|x-\xi|>2 \Delta x\}} G_{x_{i} x_{j} x_{N}}\left(x_{\theta}, \xi\right) \xi_{N}^{-\alpha}|x-\xi|^{\beta} d \xi,
$$

где $x_{\theta}$ принадлежит отрезку $[x, \bar{x}]$. Так как на множестве $|x-\xi|>2 \Delta x$ величины $|x-\xi|,|\bar{x}-\xi|$ и $\left|x_{\theta}-\xi\right|$ эквивалентны, то

$$
\begin{aligned}
\left|I_{4}\right| \leqslant C|f| & \frac{(\beta)}{\mathbb{R}_{+}^{N}} x_{N}^{\alpha}|\Delta x| \int_{B_{2 R}^{+} \cap\{|x-\xi|>2 \Delta x\}} \xi_{N}^{-\alpha}|x-\xi|^{-N-1+\beta} d \xi \\
=C|f| & \frac{(\beta)}{\mathbb{R}_{+}^{N}} x_{N}^{\alpha}|\Delta x|\left\{\int_{B_{2 R}^{+} \cap\{|x-\xi|>2 \Delta x\} \cap\left\{\xi_{N}>\varepsilon_{0} x_{N}\right\}} \xi_{N}^{-\alpha}|x-\xi|^{-N-1+\beta} d \xi\right. \\
& \left.+\int_{B_{2 R}^{+} \cap\{|x-\xi|>2 \Delta x\} \cap\left\{\xi_{N}<\varepsilon_{0} x_{N}\right\}} \xi_{N}^{-\alpha}|x-\xi|^{-N-1+\beta} d \xi\right\} \equiv I_{41}+I_{42} .
\end{aligned}
$$

Для оценки $I_{41}$ мы используем неравенство $\left\{\xi_{N}>\varepsilon_{0} x_{N}\right\}$, так что $\xi_{N}^{-\alpha} \leqslant C x_{N}^{-\alpha}$. Поэтому справедлива оценка

$$
\left|I_{41}\right| \leqslant C|f| \frac{(\beta)}{\mathbb{R}_{+}^{N}}|\Delta x| \int_{|x-\xi|>2 \Delta x}|x-\xi|^{-N-1+\beta} d \xi=C|f|_{\mathbb{R}_{+}^{N}}^{(\beta)}|\Delta x|^{\beta} .
$$

В силу (3.17) на множестве интегрирования в $I_{42}$ выполнено

$$
|x-\xi| \geqslant\left|x_{N}-\xi_{N}\right| \geqslant\left(1-\varepsilon_{0}\right) x_{N}>\frac{1-\varepsilon_{0}}{\varepsilon_{0}} \Delta x>2 \Delta x,
$$

т.е. множество интегрирования

$$
B_{2 R}^{+} \cap\{|x-\xi|>2 \Delta x\} \cap\left\{\xi_{N}<\varepsilon_{0} x_{N}\right\}=B_{2 R}^{+} \cap\left\{\xi_{N}<\varepsilon_{0} x_{N}\right\} .
$$

Кроме того, так как на этом множестве $\left|x_{N}-\xi_{N}\right| \geqslant\left(1-\varepsilon_{0}\right) x_{N}$, то в $I_{42}$

$$
|x-\xi| \geqslant \nu\left[\left(x^{\prime}-\xi^{\prime}\right)^{2}+x_{N}^{2}\right]^{1 / 2} \text {. }
$$

Поэтому

$$
\left|I_{42}\right| \leqslant C|f| \frac{(\beta)}{\mathbb{R}_{+}^{N}} x_{N}^{\alpha}|\Delta x| \int_{0}^{\varepsilon_{0} x_{N}} \xi_{N}^{-\alpha} d \xi_{N} \int_{\mathbb{R}^{N-1}} \frac{d \xi^{\prime}}{\left[\left(x^{\prime}-\xi^{\prime}\right)^{2}+x_{N}^{2}\right]^{(N+1-\beta) / 2}} .
$$

Совершая в последнем интеграле замену $x^{\prime}-\xi^{\prime}=\eta x_{N}$, имеем

$$
\begin{aligned}
\left|I_{42}\right| & \leqslant C|f| \frac{(\beta)}{\mathbb{R}_{+}^{N}} x_{N}^{\alpha}|\Delta x| x_{N}^{-N-1+\beta+N-1} \int_{0}^{\varepsilon_{0} x_{N}} \xi_{N}^{-\alpha} d \xi_{N} \\
& =C|f| \frac{(\beta)}{\mathbb{R}_{+}^{N}}|\Delta x| x_{N}^{-1+\beta}=C|f| \frac{(\beta)}{\mathbb{R}_{+}^{N}}\left(\frac{\Delta x}{x_{N}}\right)^{1-\beta}(\Delta x)^{\beta} \leqslant C|f| \frac{(\beta)}{\mathbb{R}_{+}^{N}}(\Delta x)^{\beta} .
\end{aligned}
$$


Из (3.26) и (3.28) следует, что

$$
\left|I_{4}\right| \leqslant C|f|_{\frac{\mathbb{R}_{+}^{N}}{(\beta)}}(\Delta x)^{\beta} .
$$

Собирая теперь оценки интегралов $I_{i}, i=\overline{1,4}$, получаем

$$
\left|\mathcal{A}_{2}\right| \leqslant C|f| \frac{(\beta)}{\mathbb{R}_{+}^{N}}(\Delta x)^{\beta} .
$$

Таким образом,

$$
\left|v\left(x^{\prime}, x_{N}\right)-v\left(x^{\prime}, \bar{x}_{N}\right)\right| \leqslant C|f|_{\mathbb{R}_{+}^{N}}^{(\beta)}(\Delta x)^{\beta},
$$

что дает оценку константы Гёльдера функции $v(x)$ по переменной $x_{N}$.

Оценки гладкости функции $v(x)$ по переменным $x^{\prime}$ проходят по аналогичной схеме, причем в силу (3.16) мы, как и выше, не ограничивая общности, можем считать, что $\left|x^{\prime}-\bar{x}^{\prime}\right| \leqslant \varepsilon_{0} x_{N}$. Итак, для функции $u(x)$ в $(3.3)$ доказано, что

$$
\left\langle x_{N}^{\alpha} u_{x_{i} x_{j}}\right\rangle \frac{(\beta)}{B_{R}^{+}} \leqslant C_{R}|f| \frac{(\beta)}{\mathbb{R}_{+}^{N}} .
$$

Мы доказали эту оценку для случая, когда $i$ и $j$ не равны $N$ одновременно. Оценка же (3.30) для случая $i=j=N$ следует из уравнения (3.1).

Из соотношения (3.12) и оценки (3.16) следует также, что

$$
\left|x_{N}^{\alpha} u_{x_{i} x_{j}}\right| \frac{(0)}{B_{R}^{+}} \leqslant C_{R}|f| \frac{(\beta)}{\mathbb{R}_{+}^{N}} .
$$

Кроме того, в силу ограничения $0<\beta<\min \{\alpha, 1-\alpha\}$ нормы $|D u|_{B_{R}^{+}}^{(\beta)}$ являются младшими и их оценка следует из (3.31). Действительно, покажем гёльдеровость $u_{x_{i}}, i=\overline{1, N}$, по переменной $x_{N}$ с показателем $1-\alpha$. Пусть $0<\bar{x}_{N}<x_{N}$. Тогда

$$
u_{x_{i}}\left(x^{\prime}, x_{N}\right)-u_{x_{i}}\left(x^{\prime}, \bar{x}_{N}\right)=\int_{\bar{x}_{N}}^{x_{N}} u_{x_{i} \xi_{N}}\left(x^{\prime}, \xi_{N}\right) d \xi_{N} .
$$

Отсюда и из (3.31) получаем

$$
\left|u_{x_{i}}\left(x^{\prime}, x_{N}\right)-u_{x_{i}}\left(x^{\prime}, \bar{x}_{N}\right)\right| \leqslant C|f| \frac{(\beta)}{\mathbb{R}_{+}^{N}} \int_{\bar{x}_{N}}^{x_{N}} \xi_{N}^{-\alpha} d \xi_{N} \leqslant C|f| \frac{(\beta)}{\mathbb{R}_{+}^{N}}\left|x_{N}-\bar{x}_{N}\right|^{1-\alpha} .
$$

Теперь получим оценку константы Гёльдера $u_{x_{i}}$ по переменным $x^{\prime}$ с показателем $1-\alpha$. Пусть

$$
A \equiv \frac{\left|u_{x_{i}}\left(x^{\prime}, x_{N}\right)-u_{x_{i}}\left(\bar{x}^{\prime}, x_{N}\right)\right|}{\left|x^{\prime}-\bar{x}^{\prime}\right|^{1-\alpha}} .
$$

Если $\left|x^{\prime}-\bar{x}^{\prime}\right| \leqslant x_{N}$, то для некоторого $x_{\theta}^{\prime} \in\left[x^{\prime}, \bar{x}^{\prime}\right]$ из теоремы о среднем и (3.31) следует

$$
A \leqslant\left|\nabla u_{x_{i}}\left(x_{\theta}^{\prime}, x_{N}\right)\right|\left|x^{\prime}-\bar{x}^{\prime}\right|^{\alpha} \leqslant\left|x_{N}^{\alpha} \nabla u_{x_{i}}\left(x_{\theta}^{\prime}, x_{N}\right)\right| \leqslant C|f| \frac{(\beta)}{\mathbb{R}_{+}^{N}} .
$$


Если же $\left|x^{\prime}-\bar{x}^{\prime}\right|>x_{N}$, то оценим величину $A$ так $\left(\Delta x=\left|x^{\prime}-\bar{x}^{\prime}\right|\right)$ :

$$
\begin{gathered}
A \leqslant \frac{\left|u_{x_{i}}\left(x^{\prime}, x_{N}+\Delta x\right)-u_{x_{i}}\left(x^{\prime}, x_{N}\right)\right|}{(\Delta x)^{1-\alpha}+\frac{\left|u_{x_{i}}\left(x^{\prime}, x_{N}+\Delta x\right)-u_{x_{i}}\left(\bar{x}^{\prime}, x_{N}+\Delta x\right)\right|}{(\Delta x)^{1-\alpha}}} \\
+\frac{\left|u_{x_{i}}\left(\bar{x}^{\prime}, x_{N}+\Delta x\right)-u_{x_{i}}\left(\bar{x}^{\prime}, x_{N}\right)\right|}{(\Delta x)^{1-\alpha}} \equiv I_{1}+I_{2}+I_{3} .
\end{gathered}
$$

При этом

$$
I_{1}+I_{3} \leqslant C|f| \frac{(\beta)}{\mathbb{R}_{+}^{N}}
$$

на основании (3.32), а для $I_{2}$ выполнено условие $\left|x^{\prime}-\bar{x}^{\prime}\right| \leqslant x_{N}+\Delta x$, так что по доказанному

$$
I_{2} \leqslant C|f|_{\frac{\mathbb{R}_{+}^{N}}{(\beta)}}
$$

Таким образом,

$$
\left\langle u_{x_{i}}\right\rangle \frac{(1-\alpha)}{B_{R}^{+}} \leqslant C|f| \frac{(\beta)}{\mathbb{R}_{+}^{N}} .
$$

Следовательно, нами доказано следующая лемма.

Лемма 2. Пусть в задаче (3.1), (3.2) функиия $f(x)$ финитна с носителем $B_{R}^{+}$, причем $f(x) \in C^{\beta}\left(\overline{\mathbb{R}_{+}^{N}}\right)$. Тогда $u(x) \in C_{\alpha}^{2+\beta}\left(\overline{\mathbb{R}_{+}^{N}}\right)$ и справедлива оценка

$$
|u(x)|_{\alpha, B_{R}^{+}}^{(2+\beta)} \leqslant C_{R}|f|_{\frac{\mathbb{R}_{+}^{N}}{(\beta)}} .
$$

\section{§ 4. Априорная оценка Шаудера для задачи (1.3), (1.4)}

Применяя стандартную технику оценок Шаудера (см., например, [17], [18]) и опираясь на результаты $\S 2$ и $\S 3$, для решения задачи $(1.3),(1.4)$ из класса $C_{\alpha}^{2+\beta}(\bar{\Omega})$ получаем следующую априорную оценку:

$$
|u(x)|_{\alpha, \bar{\Omega}}^{(2+\beta)} \leqslant C\left(|f|_{\bar{\Omega}}^{(\beta)}+|\varphi|_{\partial \Omega}^{(2+\beta-\alpha)}+|u|_{\bar{\Omega}}^{(0)}\right) .
$$

При этом используется доказанный в $\S 3$ факт, что для $u(x) \in C_{\alpha}^{2+\beta}(\bar{\Omega})$ справедлива оценка

$$
|\nabla u|_{\bar{\Omega}}^{(1-\alpha)} \leqslant C|u|_{\alpha, \bar{\Omega}}^{(2+\beta)} .
$$

Так как $\beta<1-\alpha$, то отсюда следует, что для любого $\varepsilon>0$ справедливо неравенство

$$
|u|_{\bar{\Omega}}^{(1+\beta)} \leqslant \varepsilon|u|_{\alpha, \bar{\Omega}}^{(2+\beta)}+C_{\varepsilon}|u|_{\bar{\Omega}}^{(0)} .
$$

Кроме того, из классического принципа максимума следует, что если $a(x) \leqslant 0$, то решение задачи (1.3), (1.4) единственно (см., например, [18], [17]). Более того, как мы покажем ниже при доказательстве оценки (4.8), в этом случае справедлива оценка

$$
|u(x)|_{\alpha, \bar{\Omega}}^{(2+\beta)} \leqslant C\left(|f|_{\bar{\Omega}}^{(\beta)}+|\varphi|_{\partial \Omega}^{(2+\beta-\alpha)}\right) .
$$


Поскольку возможно продолжение функции $\varphi(x)$ с границы $\partial \Omega$ на всю область $\bar{\Omega}$ до функции класса $C_{\alpha}^{2+\beta}(\bar{\Omega})$ с сохранением нормы, мы будем, как отмечено выше, не ограничивая общности, считать, что $\varphi(x) \equiv 0$. Кроме того, в дальнейшем мы считаем в (1.3) выполненным условие

$$
a(x) \leqslant 0 .
$$

Имея в виду доказательство разрешимости задачи (1.3), (1.4) методом продолжения по параметру, мы рассмотрим для $\omega \in[0,1]$ семейство задач

$$
\begin{gathered}
d(x)^{\alpha} \sum_{i, j=1}^{N} a_{i j} u_{x_{i} x_{j}}^{(\omega)}+\omega\left[\sum_{i=1}^{N} a_{i} u_{x_{i}}^{(\omega)}+a u^{(\omega)}\right]=f(x), \quad x \in \Omega, \\
\left.u^{(\omega)}\right|_{\partial \Omega}=0 .
\end{gathered}
$$

Отметим,что при $\omega=1$ мы получаем задачу (1.3), (1.4), а при $\omega=0$ - задачу

$$
\begin{aligned}
d(x)^{\alpha} \sum_{i, j=1}^{N} a_{i j} u_{x_{i} x_{j}} & =f(x), \quad x \in \Omega, \\
\left.u\right|_{\partial \Omega} & =0 .
\end{aligned}
$$

Так как нормы коэффициентов уравнений (4.4) равномерно по $\omega$ ограничены, то равномерно по $\omega$ справедлива оценка (4.1). При этом, так как коэффициент при $u^{(\omega)}(x)$ в (4.4) равен $\omega a(x) \leqslant 0$ и, следовательно, решение соответствующей задачи единственно, то равномерно по $\omega$ выполнено

$$
\left|u^{(\omega)}(x)\right|_{\alpha, \bar{\Omega}}^{(2+\beta)} \leqslant C|f|_{\bar{\Omega}}^{(\beta)} .
$$

Рассуждения для получения (4.8) из (4.1) стандартны и опираются на факт единственности задачи (4.4), (4.5) (см., например, [10]). Мы приведем эти рассуждения для полноты изложения.

Итак, предположим противное, а именно, что член $|u|_{\bar{\Omega}}^{(0)}$ в оценке (4.1) не может быть отброшен. Тогда существуют последовательности $\omega_{n} \in[0,1]$ и $f_{n}$, $\left|f_{n}\right|_{\frac{\beta}{\Omega}}^{(\beta)}=1$, такие, что

$$
\left|u_{n}\right|_{\frac{\Omega}{\Omega}}^{(0)} \geqslant n
$$

где $u_{n}=u^{\left(\omega_{n}\right)}$ - решение соответствующей задачи $(4.4),(4.5)$. При этом, не ограничивая общности, можно считать, что $\omega_{n} \rightarrow \omega_{0} \in[0,1]$. Рассмотрим последовательность

$$
v_{n}=\frac{u_{n}}{\left|u_{n}\right|_{\frac{(0)}{\Omega}}^{(0 .}} .
$$

Очевидно, что

$$
\left|v_{n}(x)\right|_{\alpha, \bar{\Omega}}^{(2+\beta)} \geqslant 1
$$

и в то же время в силу (4.1)

$$
\left|v_{n}(x)\right|_{\alpha, \bar{\Omega}}^{(2+\beta)} \leqslant C
$$

так как

$$
\left|v_{n}\right|_{\frac{(0)}{\Omega}}=1 .
$$


Функции $v_{n}$ удовлетворяют задаче $(4.4),(4.5)$ с $\omega=\omega_{n}$ и с правыми частями

$$
f \equiv g_{n} \equiv \frac{f_{n}}{\left|u_{n}\right|_{\bar{\Omega}}^{(0)}} .
$$

В силу (4.9) получаем

$$
\left|g_{n}\right|_{\frac{(\beta)}{\Omega}}=\frac{\left|f_{n}\right|_{\frac{(\beta)}{\Omega}}}{\left|u_{n}\right|_{\bar{\Omega}}^{(0)}} \leqslant \frac{1}{n} \rightarrow 0, \quad n \rightarrow \infty .
$$

Из (4.11) следует, что из последовательности $v_{n}$ можно выделить подпоследовательность (обозначим ее снова $v_{n}$ ), которая сходится к некоторой функции $v_{0}$ в пространстве $C_{\alpha}^{2+\beta^{\prime}}(\bar{\Omega})$ с $\beta^{\prime}<\beta$. При этом в силу $(4.10),(4.12)$ функция $v_{0}(x)$ не равна тождественно нулю и $v_{0}(x)$ удовлетворяет задаче $(4.4),(4.5)$ с $f \equiv 0$ (как следует из (4.13)) и с $\omega=\omega_{0}$. Последнее же противоречит единственности решения задачи (4.4), (4.5), что доказывает оценки (4.8) и (4.2).

Ввиду применимости (в силу (4.8)) рассуждений о разрешимости методом продолжения по параметру (см., например, [17]) достаточно доказать разрешимость в $C_{\alpha}^{2+\beta}(\bar{\Omega})$ задачи $(4.6),(4.7)$. Запишем уравнение (4.6) в виде

$$
a_{i j} u_{x_{i} x_{j}}=\frac{f(x)}{d(x)^{\alpha}} \equiv g(x), \quad x \in \Omega .
$$

Поскольку $g(x)$ в (4.14) принадлежит пространству $L_{p}(\Omega), 1<p<1 / \alpha$, то из результатов [18] следует, что задача (4.14), (4.7) (и, тем самым, задача (4.6), $(4.7))$ имеет единственное решение $u(x)$ из пространства $W_{p}^{2}(\Omega)$. Нашей задачей теперь будет показать, что это решение на самом деле принадлежит пространству $C_{\alpha}^{2+\beta}(\bar{\Omega})$.

\section{§5. Разрешимость и оценки решений задач в областях специального вида}

5.1. Простейшее уравнение в области специального вида. Пусть $\Omega_{d}=\left\{x \in \mathbb{R}^{N}:\left|x_{i}\right| \leqslant d, i=\overline{1, N-1}, 0<x_{N}<d\right\}, d \geqslant d_{0}>0$. Рассмотрим в области $\Omega_{d}$ задачу

$$
\begin{gathered}
x_{N}^{\alpha} \Delta u=f(x), \quad x \in \Omega_{d}, \\
\left.u\right|_{x_{N}=0, d}=0, \\
\left.\frac{\partial u}{\partial n}\right|_{x_{i}= \pm d}=0,
\end{gathered}
$$

где $\partial u / \partial n-$ производная по внешней нормали к границе $\Omega_{d}, f(x) \in C^{\beta}(\bar{\Omega})$.

ЗАмечАниЕ. Область $\Omega_{d}$ имеет ребра и угловые точки на своей границе. Однако это обстоятельство несущественно, так как в силу условия (5.3) решение может быть продолжено четным образом через каждую грань $x_{i}= \pm d, i=$ $\overline{1, N-1}$, области $\Omega_{d}$. Поэтому в дальнейшем мы будем проводить локальные 
оценки только вблизи регулярных точек границы области $\Omega_{d}$, считая условие (5.3) по существу условием периодичности по каждой из переменных $x_{i}$, $i=\overline{1, N-1}$.

Запишем уравнение (5.1) в виде

$$
\Delta u=g(x) \equiv \frac{f(x)}{x_{N}^{\alpha}},
$$

где $g(x) \in L_{p}\left(\Omega_{d}\right)$ с $1<p<1 / \alpha$. Из результатов [18] с учетом приведенного замечания следует, что задача (5.1)-(5.3) имеет единственное решение из пространства $W_{p}^{2}\left(\Omega_{d}\right)$.

Действительно, решение задачи (5.1)-(5.3) из $W_{p}^{2}\left(\Omega_{d}\right)$ можно получить следующим образом. Пусть $g_{\varepsilon}(x), \varepsilon>0$, есть последовательность функций класса $C^{\infty}\left(\Omega_{d}\right)$, полученных сглаживанием функции $f(x) / x_{N}^{\alpha}$, таких, что $g_{\varepsilon}(x) \equiv 0$ в окрестности границы $\Omega_{d}$ и

$$
\left\|g_{\varepsilon}(x)-\frac{f(x)}{x_{N}^{\alpha}}\right\|_{L_{p}\left(\Omega_{d}\right)} \rightarrow 0, \quad \varepsilon \rightarrow 0 .
$$

Тогда задача (5.1)-(5.3) с правой частью $g_{\varepsilon}(x)$ может быть решена методом разделения переменных, так как коэффициенты Фурье $g_{\varepsilon}^{(n)}$ функции $g_{\varepsilon}(x)$ убывают быстрее любой степени номера $n$. Это дает решение $u_{\varepsilon}(x)$ задачи $(5.1)-(5.3)$ из класса $C^{\infty}\left(\bar{\Omega}_{d}\right)$. В то же время в силу приведенного замечания для решений $u_{\varepsilon}(x)$ (как следует из [18; гл. 9]) справедлива априорная оценка

$$
\left\|u_{\varepsilon}\right\|_{W_{p}^{2}\left(\Omega_{d}\right)} \leqslant C\left\|g_{\varepsilon}(x)\right\|_{L_{p}\left(\Omega_{d}\right)} \leqslant C\left\|\frac{f(x)}{x_{N}^{\alpha}}\right\|_{L_{p}\left(\Omega_{d}\right)} .
$$

Требуемое решение из $W_{p}^{2}\left(\Omega_{d}\right)$ исходной задачи получается предельным переходом при $\varepsilon \rightarrow 0$.

Покажем, что на самом деле это решение принадлежит $C_{\alpha}^{2+\beta}\left(\bar{\Omega}_{d}\right)$. При этом достаточно рассмотреть только окрестность границы $\left\{x_{N}=0\right\}$, так как вне этой окрестности уравнение не вырождается, и в силу хорошо известных локальных оценок (см., например, [18], [17]) решение вне окрестности $\left\{x_{N}=0\right\}$ принадлежит классу $C^{2+\beta}$.

Пусть $x_{0} \in\left\{x_{N}=0\right\},\left|x_{0 i}\right|<d, i=\overline{1, N-1}$. Пусть $\eta_{r}(x)$ - гладкая срезающая функция такая, что $\eta_{r}(x) \equiv 1$ на $B_{r}=B_{r}\left(x_{0}\right)=\left\{\left|x-x_{0}\right| \leqslant r\right\}$ и $\eta_{r}(x) \equiv 0$ вне $B_{2 r}=B_{2 r}\left(x_{0}\right), 2 r<\min _{i}\left\{d-\left|x_{0 i}\right|\right\}$.

Умножим уравнение $(5.1)$ на $\eta_{r}(x)$. Получим

$$
x_{N}^{\alpha} \Delta v_{r}=\left[\eta_{r} f(x)\right]+x_{N}^{\alpha}\left[2 \nabla \eta_{r} \nabla u(x)+u(x) \Delta \eta_{r}\right] \equiv g_{1}(x)+x_{N}^{\alpha} g_{2}(x),
$$

где $v_{r}(x)=u(x) \eta_{r}(x)$ - финитная функция. $\mathrm{B}$ соответствии с разбиением правой части в (5.5) представим $v_{r}$ в виде суммы функций $v_{r 1}$ и $v_{r 1}$, т.е. $v_{r}=v_{r 1}+v_{r 2}$, где $v_{r 1}$ и $v_{r 1}$ удовлетворяют условиям

$$
\begin{gathered}
x_{N}^{\alpha} \Delta v_{r 1}=g_{1}(x), \quad x \in\left\{x_{N}>0\right\},\left.\quad v_{r 1}\right|_{x_{N}=0}=0, \\
\Delta v_{r 2}=g_{2}(x), \quad x \in\left\{x_{N}>0\right\},\left.\quad v_{r 2}\right|_{x_{N}=0}=0,
\end{gathered}
$$


причем $g_{1}, g_{2}$ финитны. А поскольку $u(x) \in W_{p}^{2}\left(\Omega_{d}\right)$, то в силу теорем вложения $g_{2}(x) \in L_{p_{1}}\left(\mathbb{R}_{+}^{N}\right)$, если $p<N$, где $p_{1}=N p /(N-p)\left(\right.$ так как $\left.\nabla u \in L_{p_{1}}\left(\mathbb{R}_{+}^{N}\right)\right)$; если же $p>N$, то из теорем вложения следует, что $g_{2} \in C^{\delta}\left(\overline{\mathbb{R}_{+}^{N}}\right)$ с некоторым $\delta>0$.

Применение результатов $\S 3$ дает $v_{r 1} \in C_{\alpha}^{2+\beta}\left(B_{2 r}^{+}\right)$, а из [18; гл. 9] следует $v_{r 2} \in W_{p_{1}}^{2}\left(B_{2 r}^{+}\right)$. Если $p_{1}>N$, то $\nabla v_{r 2} \in C^{\delta}\left(B_{2 r}^{+}\right)$с некоторым $\delta>0$, если же $p_{1}<N$, то $\nabla v_{r 2} \in L_{p_{2}}\left(B_{2 r}^{+}\right), p_{2}=N p_{1} /\left(N-p_{1}\right)$. Таким образом, если $p_{1}<N$, то $\nabla v_{r}=\nabla v_{r 1}+\nabla v_{r 2} \in L_{p_{2}}\left(B_{2 r}^{+}\right)$. То есть $\nabla u$ на множестве $B_{r}^{+}$принадлежит пространству $L_{p_{2}}\left(B_{r}^{+}\right)$или даже $C^{\delta}\left(B_{r}^{+}\right)\left(\right.$если $\left.p_{1}>N\right)$.

Умножая уравнение (5.1) на функцию $\eta_{r / 2}(x)$ и повторяя предыдущие рассуждения, получаем, что $u(x) \in W_{p_{2}}^{2}\left(B_{r / 2}^{+}\right)$, и либо $\nabla u \in C^{\delta}\left(B_{r / 2}^{+}\right)$, если $p_{2}>N$, либо $\nabla u \in L_{p_{3}}\left(B_{r / 2}^{+}\right)$, где $p_{3}=N p_{2} /\left(N-p_{2}\right)$. Продолжая этот процесс, мы через конечное число шагов получим, что $p_{k}>N$, и, следовательно, решение $u(x)$ принадлежит пространству $W_{p_{k}}^{2}\left(B_{r / 2^{k}}^{+}\right)$и по вложению имеет гладкий градиент $\nabla u \in C^{\delta}\left(B_{r / 2^{k}}^{+}\right), \delta>0$. Срезая $u(x)$ внутри $B_{r / 2^{k}}^{+}$срезкой $\eta_{r / 2^{k+1}}$, приходим к соотношению вида $(5.5)$, в котором $g_{2} \in C^{\delta}\left(B_{r / 2^{k+1}}^{+}\right)$. Следовательно, на $B_{r / 2^{k+1}}^{+}$решение $u(x)$ представимо в виде $u=u_{1}+u_{2}$, где $u_{1} \in C_{\alpha}^{2+\beta}\left(B_{r / 2^{k+1}}^{+}\right), u_{2} \in C^{2+\delta}\left(B_{r / 2^{k+1}}^{+}\right)$. Поэтому на этом множестве $\nabla u=$ $\nabla u_{1}+\nabla u_{2} \in C^{1-\alpha}\left(B_{r / 2^{k+1}}^{+}\right)$. Наконец, производя последнюю срезку внутри $B_{r / 2^{k+1}}^{+}$, из соотношения вида (5.5) получаем, что $u(x) \in C_{\alpha}^{2+\beta}\left(B_{r / 2^{k+2}}^{+}\right)$, так как $u=u_{1}+u_{2}$, где $u_{1} \in C_{\alpha}^{2+\beta}\left(B_{r / 2^{k+2}}^{+}\right)$, а $u_{2} \in C^{2+\beta}\left(B_{r / 2^{k+2}}^{+}\right)$.

Следовательно, полученное решение из $W_{p}^{2}\left(\Omega_{d}\right)$ задачи (5.1)-(5.3) на самом деле принадлежит пространству $C_{\alpha}^{2+\beta}\left(\bar{\Omega}_{d}\right)$, что доказывает следующую лемму.

Лемма 3. Задача (5.1)-(5.3) имеет единственное решение и(x) из класса $C_{\alpha}^{2+\beta}\left(\bar{\Omega}_{d}\right)$, причем

$$
|u(x)|_{\alpha, \overline{\Omega_{d}}}^{(2+\beta)} \leqslant C|f(x)|_{\Omega_{d}}^{(\beta)} .
$$

5.2. Более общее уравнение в специальной области. Пусть $F\left(x^{\prime}\right) \in$ $C^{2+\beta}\left(\bar{\Pi}_{d}\right)$, где $\Pi_{d}=\left\{x^{\prime}:\left|x_{i}\right|<d, i=\overline{1, N-1}\right\},\left|F\left(x^{\prime}\right)\right| \leqslant d / 2$, и при каждом $i=\overline{1, N-1}$ выполнено $\nabla F\left(x^{\prime}\right)=0$ при $\left|x_{i}\right|=d$. Обозначим $\Omega_{F}=\left\{x \in \mathbb{R}^{N}\right.$ : $\left.x^{\prime} \in \Pi_{d}, F\left(x^{\prime}\right)<x_{N}<d+F\left(x^{\prime}\right)\right\}, \Gamma_{F}=\left\{x: x_{N}=F\left(x^{\prime}\right), x^{\prime} \in \Pi_{d}\right\}$. Рассмотрим в области $\Omega_{F}$ задачу определения функции $u(x)$ по условиям

$$
\begin{gathered}
d(x)^{\alpha} L u \equiv d(x)^{\alpha} \sum_{i, j=1}^{N} a_{i j}(x) u_{x_{i} x_{j}}(x)=f(x), \quad x \in \Omega_{F}, \\
u(x)=0 \quad \text { при } x_{N}=F\left(x^{\prime}\right) \quad \text { и при } x_{N}=d+F\left(x^{\prime}\right), \\
\frac{\partial u}{\partial n}=0 \quad \text { при каждом } i=\overline{1, N-1} \quad \text { при }\left|x_{i}\right|=d,
\end{gathered}
$$

где свойства $d(x)$ и $a_{i j}(x)$ такие же, как в задаче $(1.2)-(1.6)$, в частности

$$
\nu \operatorname{dist}\left(x, \Gamma_{F}\right) \leqslant d(x) \leqslant C \operatorname{dist}\left(x, \Gamma_{F}\right) .
$$


Наряду с уравнением (5.9) рассмотрим также уравнение специального вида

$$
\begin{array}{r}
\left(x_{N}-F\left(x^{\prime}\right)\right)^{\alpha} L^{*} u \equiv\left(x_{N}-F\left(x^{\prime}\right)\right)^{\alpha}\left[\Delta u+2 \sum_{i, j=1}^{N-1} F_{x_{i}}\left(x^{\prime}\right) \frac{\partial^{2} u}{\partial x_{i} \partial x_{N}}\right. \\
\left.+\left(\nabla_{x^{\prime}} F\right)^{2} \frac{\partial^{2} u}{\partial x_{N}^{2}}+\Delta_{x^{\prime}} F\left(x^{\prime}\right) \frac{\partial u}{\partial x_{N}}\right]=f(x) .
\end{array}
$$

Оператор уравнения (5.13) подобран таким образом, что при замене

$$
y_{N}=x_{N}-F\left(x^{\prime}\right), \quad y^{\prime}=x^{\prime}
$$

уравнение (5.13) в области $\Omega_{F}$ переходит в точности в уравнение $(5.1)$ в области $\Omega_{d}$, причем функция $\widehat{d}(x)=x_{N}-F\left(x^{\prime}\right)$ удовлетворяет (5.12). Граничные же условия сохраняются в силу свойства $\nabla_{x^{\prime}} F=0$ при $\left|x_{i}\right|=d$ функции $F\left(x^{\prime}\right)$.

Таким образом, задача $(5.13),(5.10),(5.11)$ имеет единственное решение, для которого справедлива оценка (5.8) (где константа $C$ зависит от $F$ ).

Рассмотрим семейство уравнений (т.е. применим метод продолжения по параметру)

$$
L_{\omega} u \equiv\left[(1-\omega)\left(x_{N}-F\left(x^{\prime}\right)\right)+\omega d(x)\right]^{\alpha}\left[(1-\omega) L^{*}+\omega L\right] u_{\omega}(x)=f(x)
$$

с граничными условиями (5.10), (5.11).

Точно так же, как была получена оценка (4.8), для задачи (5.15), (5.10), (5.11) справедлива равномерная по $\omega$ априорная оценка

$$
\left|u_{\omega}(x)\right|_{\alpha, \overline{\Omega_{F}}}^{(2+\beta)} \leqslant C|f(x)| \frac{(\beta)}{\Omega_{F}} .
$$

Поскольку при $\omega=0$ задача (5.15), (5.10), (5.11) имеет единственное решение и равномерно по $\omega$ выполнена оценка (5.16), то стандартные рассуждения метода продолжения по параметру показывают, что справедлива следующая лемма.

Лемма 4. Задача (5.9)-(5.11) имеет единственное решение и(x) из пространства $C_{\alpha}^{2+\beta}\left(\bar{\Omega}_{F}\right)$, для которого справедлива оценка

$$
|u(x)|_{\alpha, \overline{\Omega_{F}}}^{(2+\beta)} \leqslant C|f(x)|_{\frac{\Omega_{F}}{(\beta)}} .
$$

\section{§ 6. Завершение доказательства теоремы 1}

Как было отмечено в конце $\S 4$, задача (4.6), (4.7) имеет единственное решение из пространства $W_{p}^{2}(\Omega)$. Покажем, что на самом деле это решение принадлежит пространству $C_{\alpha}^{2+\beta}(\bar{\Omega})$. При этом достаточно рассмотреть только окрестность границы $\partial \Omega$.

Пусть $x_{0} \in \partial \Omega$, и пусть $\lambda>0$ настолько мало, что участок границы $\partial \Omega \cap$ $B_{5 \lambda\left(x_{0}\right)}$ в местных координатах, связанных с точкой $x_{0}$ (см. [13]), представим в виде

$$
y_{N}=\Phi\left(y^{\prime}\right), \quad \Phi\left(y^{\prime}\right) \in C^{2+\beta}\left(\left\{\left|y^{\prime}\right| \leqslant 5 \lambda\right\}\right) .
$$


Пусть $\eta_{\lambda}(x) \equiv 1$ в $B_{\lambda}\left(x_{0}\right)$ и $\eta_{\lambda}(x) \equiv 0$ вне $B_{2 \lambda}\left(x_{0}\right)$ - гладкая срезающая функция. Умножая уравнение $(4.6)$ на $\eta_{\lambda}(x)$, получим

$$
\begin{aligned}
d(x)^{\alpha} \sum_{i, j=1}^{N} a_{i j} v_{x_{i} x_{j}} & =f \eta_{\lambda}+d(x)^{\alpha} \sum_{i, j=1}^{N}\left[a_{i j} u_{x_{i}} \eta_{x_{j}}+a_{i j} \eta_{x_{i} x_{j}} u\right] \\
& \equiv g_{1}(x)+d(x)^{\alpha} g_{2}(x),
\end{aligned}
$$

где $v(x)=u(x) \eta_{\lambda}(x)$ и $v(x)$ вместе с $g_{1}, g_{1}$ отличны от нуля только на множестве $\Omega_{2 \lambda}=\Omega \cap B_{2 \lambda}\left(x_{0}\right)$.

Пусть $\zeta\left(y^{\prime}\right)$ - гладкая срезающая функция на $\left\{\left|y^{\prime}\right| \leqslant 5 \lambda\right\}$ такая, что $\zeta\left(y^{\prime}\right) \equiv 1$ при $\left|y^{\prime}\right| \leqslant 3 \lambda$ и $\zeta\left(y^{\prime}\right) \equiv 0$ при $\left|y^{\prime}\right| \geqslant 4 \lambda$. Обозначим

$$
F\left(y^{\prime}\right)=\Phi\left(y^{\prime}\right) \zeta\left(y^{\prime}\right)
$$

Пусть еще $\theta\left(y^{\prime}\right)$ - гладкая функция такая, что $\theta\left(y^{\prime}\right) \equiv 1$ при $\left|y^{\prime}\right| \leqslant 2 \lambda$ и $\theta\left(y^{\prime}\right) \equiv 0$ при $\left|y^{\prime}\right| \geqslant 3 \lambda$. Обозначим в местных координатах

$$
\begin{gathered}
\widetilde{d}(y)=\theta\left(y^{\prime}\right) d(x(y))+\left[1-\theta\left(y^{\prime}\right)\right]\left[y_{N}-F\left(y^{\prime}\right)\right], \quad \widetilde{d}(x) \equiv \widetilde{d}(y(x)), \\
\widetilde{a}_{i j}(y)=\theta\left(y^{\prime}\right) a_{i j}(x(y))+\left[1-\theta\left(y^{\prime}\right)\right] \delta_{i j}, \quad \widetilde{a}_{i j}(x) \equiv \widetilde{a}_{i j}(y(x)) .
\end{gathered}
$$

Тогда функция $v(x)$ удовлетворяет в области $\Omega_{F}=\left\{y:\left|y_{i}\right|<5 \lambda, i=\overline{1, N-1}\right.$, $\left.F\left(y^{\prime}\right)<y_{N}<5 \lambda+F\left(y^{\prime}\right)\right\}$ уравнению

$$
\widetilde{d}(x)^{\alpha} \widetilde{a}_{i j} v_{x_{i} x_{j}}=g_{1}(x)+\widetilde{d}(x)^{\alpha} g_{2}(x),
$$

где $g_{1}(x) \in C^{\beta}\left(\bar{\Omega}_{F}\right), g_{2}(x) \in L_{p_{1}}\left(\Omega_{F}\right), p_{1}=N p /(N-p)$.

Опираясь теперь на результаты $\S 5$ и повторяя рассуждения из п. 5.1 при доказательстве леммы 3 , мы через конечное число шагов получим, что $u(x) \in$ $C_{\alpha}^{2+\beta}\left(\bar{\Omega} \cap B_{r}\left(x_{0}\right)\right)$ с достаточно малым $r>0$. Таким образом, задача (4.6), (4.7), а тем самым, и задача $(1.3),(1.4)$ при условии (4.3) имеет единственное решение $u(x)$ из класса $C_{\alpha}^{2+\beta}(\bar{\Omega})$, для которого справедлива оценка (1.10).

Пусть теперь коэффициент $a(x)$ в уравнении (1.3) имеет произвольный знак. Запишем уравнение (1.9) в виде

$$
\left(L u-\lambda_{0} u\right)-\left(\lambda-\lambda_{0}\right) u=f,
$$

где $\lambda_{0}$ вещественно и настолько велико, что $a(x)-\lambda_{0} \leqslant 0$ в $\bar{\Omega}$. Тогда уравнению (6.6) можно придать вид

$$
\left[I-\left(\lambda-\lambda_{0}\right)\left(L-\lambda_{0}\right)^{-1}\right] u=\left(L-\lambda_{0}\right)^{-1} f \equiv g,
$$

где $g \in C_{\alpha}^{2+\beta}(\bar{\Omega})$, а оператор $\left(L-\lambda_{0} I\right)^{-1}$ вполне непрерывен на $C^{\beta}(\bar{\Omega})$.

Поскольку для уравнения (6.7) справедлива теория Фредгольма, это завершает доказательство теоремы 1.

\section{Список литературы}

[1] О.А. Олейник, Е. В. Радкевич, "Уравнения второго порядка с неотрицательной характеристической формой”, Итоги науки. Сер. Математика. Мат. анал. 1969, ВИНИТИ, М., 1971, 7-252. 
[2] М.В. Келдыш, "О некоторых случаях вырождения уравнения эллиптического типа на границе области", ДАН СССР, 77:2 (1951), 181-183.

[3] G. Fickera, "Sulle equazioni differenziali lineari ellittico-paraboliche del secondo ordine", Atti Accad. Naz. Lincei. Mem. Cl. Sci. Fis. Mat. Nat. Sez. I (8), 5:1 (1956), $3-30$.

[4] М.И. Вишик, "Краевые задачи для эллиптических уравнений, вырождающихся на границе области", Матем. сб., 35(77):3 (1954), 513-568; англ. пер.: M. Visik, "Boundary-value problems for elliptic equations degenerating on the boundary of a region", Amer. Math. Soc. Transl. Ser. 2, 35 (1964), 15-78.

[5] М.И. Вишик, О.А. Ладыженская, "Краевые задачи для уравнений в частных производных и некоторых классов операторных уравнений", УМH, 11:6 (1956), 41-97; англ. пер.: M. I. Vishik, O. A. Ladyzhenskaya, "Boundary value problems for partial differential equations and certain classes of operator equations", Amer. Math. Soc. Transl. Ser. 2, 10 (1958), 223-281.

[6] О.А. Олейник, "О линейных уравнениях второго порядка с неотрицательной характеристической формой", Матем. сб., 69(111):1 (1966), 111-140.

[7] М. И. Вишик, В.В.Грушин, "Краевые задачи для эллиптических уравнений, вырождающихся на границе области", Матем. сб., 80(122):4(12) (1969), 455-491; англ. пер.: M. I. Višik, V. V. Grušin, "Boundary value problems for partial differential equations and certain classes of operator equations jour Math. USSR-Sb.", 9:4 (1969), 423-454.

[8] О.А. Олейник, Е. В. Радкевич, Уравнения с неотрицателъной характеристической формой, Изд-во Моск. ун-та, М., 2010.

[9] S. Levendorskii, Degenerate elliptic equations, Math. Appl., 258, Kluwer Acad. Publ., Dordrecht, 1993.

[10] C. Goulaouic, N. Shimakura, "Regularié hölderienne de certains problèmes aux limites elliptiques dégénérés", Ann. Scuola Norm. Sup. Pisa Cl. Sci. (4), 10:1 (1983), 79-108.

[11] Б. В. Базалий, С. П. Дегтярев, "Об одной граничной задаче для сильно вырождающегося эллиптического уравнения второго порядка в угловой области”, Укр. матем. журн., 59:7 (2007), 867-883; англ. пер.: B. V. Bazalii, S. P. Degtyarev, "On a boundary value problem for a strongly degenerate second order elliptic equation in an angular domain", Ukrainian Math. J., 59:7 (2007), 955-975.

[12] С.П. Дегтярев, “Об оптимальной регулярности решений первой краевой задачи для одного класса вырождающихся эллиптических уравнений”, Укр. матем. вісник, 3:4 (2006), 443-466; англ. пер.: S. P. Degtyarev, "On the optimal regularity of solutions of the first boundary value problem for a class of degenerate elliptic equations", Ukr. Math. Bull., 3:4 (2006), 423-446.

[13] О.А. Ладыженская, В.А. Солонников, Н.Н. Уральцева, Линейные и квазилинейные уравнения параболического типа, Наука, М., 1967; англ. пер.: O. A. Ladyzhenskaya, V. A. Solonnikov, N. N. Ural'tseva, Linear and quasi-linear equations of parabolic type, Transl. Math. Monogr., 23, Amer. Math. Soc., Providence, RI, 1968.

[14] И. М. Стейн, Сингулярные интегралы и дифференциалъные свойства функций, Мир, М., 1973; пер. с англ.: Е. М. Stein, Singular integrals and differentiability properties of functions, Princeton Math. Ser., 30, Princeton Univ. Press, Princeton, NJ, 1970.

[15] A. Lunardi, Analytic semigroups and optimal regularity in parabolic problems, Progr. Nonlinear Differential Equations Appl., 16, Birkhäuser, Basel, 1995.

[16] К. К. Головкин, "Об эквивалентных нормировках дробных пространств”, Работы по автоматическому программированию, численным методам и функииональному анализу, Тр. МИАН СССР, 66, Изд-во АН СССР, М.-Л., 1962, 364-383. 
[17] О.А. Ладыженская, Н. Н. Уральцева, Линейные и квазилинейные уравнения эллиптического типа, 2-е изд., Наука, M., 1973; англ. пер. 1-го изд.: О. А. Ladyzhenskaya, N. N. Ural'tseva, Linear and quasilinear elliptic equations, Math. Sci. Engrg., 46, Academic Press, New York-London, 1968.

[18] Д. Гилбарг, Н. С. Трудингер, Эллиптические дифференциалъные уравнения с частными производными второго порядка, Наука, М., 1989; пер. с англ.: D. Gilbarg, N.S. Trudinger, Elliptic partial differential equations of second order, Grundlehren Math. Wiss., 224, Springer-Verlag, Berlin, 1983.

Б. В. Базалий (В. V. Bazaliy)

Институт прикладной математики и механики

НАН Украины, г. Донецк

E-mail: bazaliy@iamm.ac.donetsk.ua

C. П. Дегтярев (S.P. Degtyarev)

Институт прикладной математики и механики

НАН Украины, г. Донецк

E-mail: degtyar@i.ua
Поступила в редакцию 18.10.2011 и 15.07 .2012 